\title{
Habilidades sociais no esporte: uma revisão de escopo
}

\author{
Social skills in sport: a scope review \\ Habilidades sociales en el deporte: una revisión de alcance
}

\section{Resumo}

Essa pesquisa trata-se de um protocolo de revisão de escopo, que tem o objetivo de explorar na literatura disponível, como as habilidades sociais são abordadas no esporte, e as suas possíveis influências na adaptação cognitiva afetiva e emocional do atleta. Para isso, serão utilizadas as bases de dados PubMed, Web of Science, Scopus e Pepsic/Lilacs, utilizando o mnemônico PCC (População, Contexto e Conceito), os termos selecionados foram, atletas, habilidades sociais e esporte, sendo interligados pelo operador booleano AND. Será considerada a literatura dos últimos 5(cinco) anos, nos idiomas português, espanhol e inglês. Para a sua construção e estruturação metodológica serão considerados os tópicos: tipo de estudo; identificação da questão de pesquisa; identificação de estudos relevantes; seleção de estudos; mapeamento da informação; apresentação dos resultados e aspectos éticos. As informações relevantes serão armazenadas no aplicativo Excel, para a análise e elaboração de tabelas e outros recursos gráficos para a exposição dos dados, resultados e posteriores discussões.

Palavras-chave: Atletas; Habilidades sociais; Esporte.

\begin{abstract}
This research is a scoping review protocol, which aims to explore in the available literature, how social skills are approached in sport, and their possible influences on the athlete's affective and emotional cognitive adaptation. For this, PubMed, Web of Science, Scopus and Pepsic / Lilacs databases will be used, using the mnemonic PCC (Population, Context and Concept), the selected terms were, athletes, social skills and sport, being interconnected by the Boolean operator E. Literature from the last 5 (five) years will be considered, in Portuguese, Spanish and English. For its construction and methodological structuring considered considered the following: kind of study; identification of the research question; identification of relevant studies; selection of studies; information mapping; presentation of results and ethical aspects. Relevant information will be stored in the Excel application, for the analysis and preparation of tables and other graphic resources for displaying data, results and subsequent results.
\end{abstract}

Keywords: Athletes; Social skills; Sport.

\section{Resumen}

Esta investigación el protocolo de revisión de alcance, que tiene como objetivo explorar la literatura disponible, cómo las habilidades sociales se acercan al deporte y sus posibles influencias en la adaptación cognitiva afectiva y emocional del deportista. Para ello se utilizarán las bases de datos PubMed, Web of Science, Scopus y Pepsic / Lilacs, utilizando el mnemónico PCC (Population, Context and Concept), los términos seleccionados fueron, deportistas, habilidades sociales y deporte, estando interconectados por el operador booleano E Se considerará la literatura de los últimos 5 (cinco) años, en portugués, español e inglés. Para su construcción y estructuración metodológica se consideró lo siguiente: tipo de estudio; identificación de la pregunta de investigación; identificación de estudios relevantes; selección de estudios; mapeo de información; presentación de resultados y aspectos éticos. La información relevante será 
almacenada en la aplicación Excel, para el análisis y elaboración de tablas y otros recursos gráficos para la visualización de datos, resultados y resultados posteriores.

Palabras clave: Atletas; Habilidades sociales; Deporte.

\section{Introdução}

A Psicologia do Esporte é um campo de conhecimento que tem como objetivo ensinar estratégias comportamentais e cognitivas para o atleta utilizar no seu cotidiano que irão otimizar o seu rendimento em treinos e competições (Medeiros \& Lacerda, 2017). Considerando que as práticas esportivas envolvem diversas funções e habilidades cognitivas, físicas e motoras, sendo construídas e aprimoradas durante essa atividade física.

Todas as atividades esportivas possuem regras e formas de execução em diferentes ambientes e o desempenho atlético irá depender da habilidade do atleta em empregar os seus recursos cognitivos, emocionais e afetivos de forma a obter o melhor resultado no contexto esportivo. Essa habilidade depende da relação de diversos fatores psicológicos, dentre eles destaca-se, a personalidade (Filho et al., 2015; Santos et al., 2011), inteligência (Braga et al., 2007; García-Coll et al., 2013) e habilidades sociais (Bueno et al., 2011; Cruz \& Sehnem, 2015; Rocha \& Monteiro, 2017; Fernandes et al., 2019).

O construto utilizado nessa pesquisa são as Habilidades Sociais, que se refere à capacidade do indivíduo de articular pensamentos, sentimentos, ações e atitudes em busca de um objetivo (Bueno et al., 2011; Feitosa et al., 2005; Freitas et al., 2016). De outro modo, "é um construto descritivo dos comportamentos sociais valorizados em uma determinada cultura com alta probabilidade de resultados favoráveis do indivíduo, seu grupo e comunidade que podem contribuir para um desempenho socialmente competente em tarefas interpessoais" (Del Prette \& Del Prette, 2018, p.24).

Pesquisas sobre o desenvolvimento de habilidades sociais em vários contextos apontam uma significativa relação entre o desenvolvimento delas e o sucesso acadêmico, afetivo e profissional (Bartholomeu et al., 2017; Cook et al., 2018; Del Prette \& Del Prette, 2017). A sua escolha como objeto desse estudo se deve à sua importância na adaptação do atleta ao contexto esportivo, principalmente através dos programas de treinamento de habilidades psicológicas (Cruz \& Sehnem, 2015; Rocha \& Monteiro, 2017; Stefanello, 2007; Weenberg \& Gould, 2017).

As habilidades sociais evidenciam-se como auxílio valoroso relacionado em prevenir comportamentos vistos como problemáticos e antissociais. A construção de repertório de habilidades sociais pode resultar em interações e estabelecimento de relações mais saudáveis, com organização psicossocial e uma prospecção de vida mais positiva. Elas oferecem vantagens em diferentes áreas de atuação do psicólogo esportivo seja para finalidades recreativas, educacionais ou rendimento.

Apesar da grande contribuição dos estudos sobre as habilidades sociais no contexto esportivo, existem poucas revisões de literatura relacionada ao tema. Na pesquisa piloto realizada pelos autores em quatro bases (Google acadêmico, SCOPUS, Web of Science e PubMed) em português, inglês e espanhol foram encontradas oito revisões de literatura relacionadas a temas, como vigorexia (Camargo et al., 2008), emoções (Trevelin \& Alves, 2019), lesões (Carter et al., 2020), estresse/ansiedade (Andreoli et al., 2020), desenvolvimento psicológico (Michael Normand et al., 2017) e futebol (Cunha et al., 2021). As revisões encontradas sobre habilidades sociais eram abordadas pela perspectiva educacional (Bessa et al., 2019, 2021). Observa-se que as revisões se tornam mais raras quando envolvem o esporte de alto rendimento.

Por isso, essa pesquisa tem como objetivo identificar na literatura como as habilidades sociais têm sido investigadas no esporte, os instrumentos/técnicas voltados para essa área e os resultados alcançados em diferentes modalidades esportivas. Vale ressaltar que dependendo da modalidade (coletiva ou individual) e do esporte investigado pode-se variar os tipos de habilidades sociais investigadas e almejadas nos atletas.

A presente revisão de escopo direciona-se a identificar por meio de uma pesquisa exploratória, aspectos relativos aos elementos principais elencados no mnemônico PCC, População: Atletas; Conceito: Habilidades Sociais e Contexto: Esporte. 
Desse modo, busca-se realizar uma pesquisa abrangente para mapear as pesquisas sobre os temas, com o propósito de conhecer, sistematizar o conhecimento e apontar lacunas existentes na abordagem do tema.

\section{Metodologia}

\subsection{Tipo de estudo}

Este estudo utiliza a metodologia orientada pelo Instituto Joanna Briggs (JBI) (Aromaratis \& Munn, 2020) e o protocolo foi elaborado com base na extensão PRISMA revisão de escopo (Tricco et al., 2018), a primeira etapa da revisão consiste na criação do protocolo, para garantir o rigor técnico e permitir a replicação das etapas utilizadas, tornando assim o processo consistente e homogêneo entre os revisores. Para garantir a transparência e a visibilidade do processo de revisão o protocolo será registrado no Open Science Framework (OSF).

Com o objetivo de investigar maiores informações sobre qual a relação entre habilidades socias no contexto do esporte. Admitindo seis estágios na sua construção e estruturação metodológica do estudo, estes são: (1) identificação da questão de pesquisa; (2) identificação de estudos relevantes; (3) seleção dos estudos relevantes; (4) mapeamento da informação; (5) apresentação dos resultados e (6) aspectos éticos.

\subsection{Identificação da questão de pesquisa}

Para obter uma visão geral da literatura, o objetivo geral da revisão de escopo será mapear as publicações disponíveis sobre habilidades sociais de atletas no contexto esportivo. Os objetivos específicos serão: identificar as teorias, formas de avaliação (técnicas e instrumentos) e os principais resultados encontrados sobre o tema.

Para definição da questão do estudo será utilizada a estrutura mnemônica: População, Contexto e Conceito (PCC), para estes foram definidos os seguintes aspectos de interesse do estudo: População (P): atletas e desportistas profissionais; Conceito (C): habilidades sociais e Contexto (C): esporte. A partir desses determinantes elaborou-se a seguinte questão norteadora: Quais são as evidências publicadas sobre habilidades sociais de atletas no esporte?

Além da pergunta principal, considerando o objetivo específico formulou-se quatro questões secundárias:

- Quais são as teorias e critérios utilizados para investigar habilidades sociais no contexto esportivo?

- As habilidades sociais são investigadas nos diferentes tipos de modalidade (individual e coletiva)?

- As habilidades sociais investigadas variam de acordo com o tipo de esporte estudado?

- Quais são as técnicas e instrumentos mais utilizados para levantar/avaliar habilidades sociais no esporte?

\subsection{Identificação dos estudos relevantes}

Foi selecionado para cada item da estratégia PCC um conjunto de descritores disponíveis nos Descritores de Ciências da Saúde (DeCS) (Figura 1). A estratégia de busca foi elaborada por meio da combinação do acrônimo PCC em três línguas diferentes (português, espanhol e inglês), considerando a aplicação dos operadores booleanos: E/OU; AND/OR e o cruzamento dos descritores, adaptados dependendo do idioma e das particularidades das seguintes bases utilizadas: Pubmed, Web of Science, SCOPUS, Literatura Latino Americana e do Caribe em Ciências da Saúde (LILACS) e as 10 (dez) primeiras páginas do Google acadêmico. Os asteriscos $\left(^{*}\right)$ serão utilizados para busca de variações linguísticas ou com múltiplos caracteres.

A elaboração das estratégias de busca foi conduzida por meio de testes das palavras-chave nas referidas bases de dados, e em consenso com todos os autores da pesquisa. Os resultados serão armazenados em uma tabela Excel, com todos os dados relevantes a condução da revisão de escopo. 
Figura 1. Termos empregados na estratégia de busca (português, inglês e espanhol).

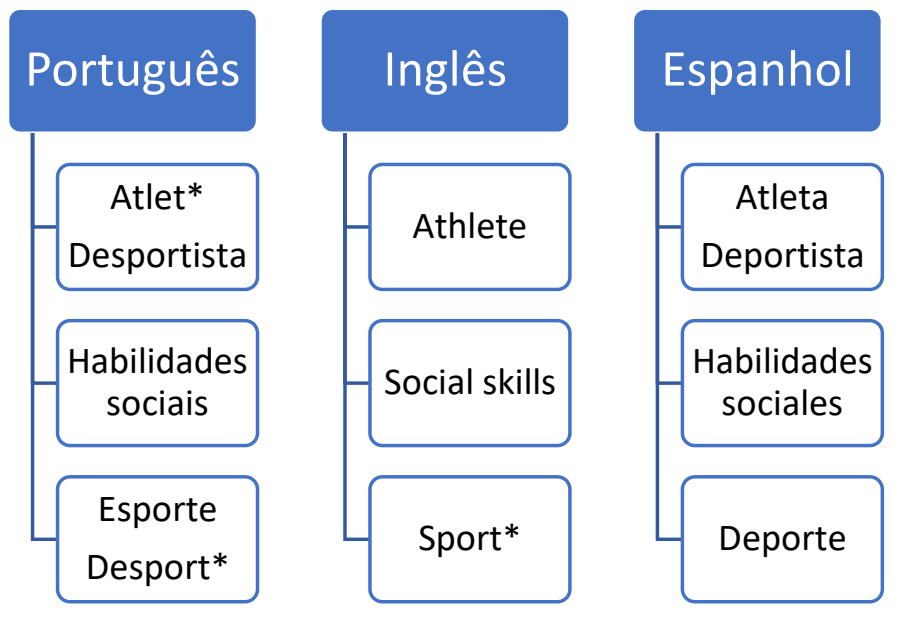

Fonte: Autores (2021).

Será feita a consulta das referências, a partir do título e resumo, dos estudos selecionados para leitura do texto completo, a fim de recuperar referências que não foram identificadas na busca inicial. Após a realização das buscas, os textos completos serão exportados para o Mendeley, tendo em vista excluir as duplicações do próprio sistema. A sistematização das fases da busca, identificação e seleção serão representadas através do Fluxograma Prisma.

\subsection{Seleção dos estudos relevantes}

A seleção dos artigos ocorrerá em três etapas: inicialmente será realizada a leitura do título e resumo para incluir estudos que respondem o objetivo da pesquisa; posteriormente os estudos selecionados serão lidos na íntegra e as referências deles serão avaliadas, para captar e incluir estudos que não foram recuperados durante a busca nas bases de dados selecionadas, e por fim será feita a leitura completa dos artigos incluídos.

Cada etapa está descrita na sequência, visando manter o rigor e a transparência a ser aplicada durante todo o processo de seleção. A primeira etapa será feita por um único revisor, o qual realizará a leitura do título e do resumo, seguindo os critérios de inclusão e exclusão, com o auxílio de um checklist (Quadro 1). Esse instrumento será aplicado para cada estudo nesta primeira fase.

A leitura completa dos artigos incluídos na primeira etapa, será realizada por três revisores de forma independente. Na segunda etapa, eles terão a responsabilidade de verificar se o artigo atende aos critérios de elegibilidade e se os mesmos respondem ao objetivo do estudo. Após a confirmação de inclusão do artigo, os revisores farão a busca dos estudos potencialmente úteis nas referências dele e todas as exclusões realizadas nesta fase serão justificadas. As discordâncias entre os revisores serão avaliadas ao final por um quarto revisor. Na última fase da revisão, todos os estudos incluídos serão divididos entre os três revisores, esses irão preencher a matriz de síntese da extração de dados. 
Quadro 1. Critérios de triagem dos estudos, segundo os critérios de inclusão e exclusão.

\begin{tabular}{|l|}
\hline \multicolumn{1}{|c|}{ Justificativa de inclusão } \\
\hline $\begin{array}{l}\text { Estudos que utilizem técnicas ou instrumentos para identificar, levantar ou avaliar habilidades sociais } \\
\text { no esporte. }\end{array}$ \\
\hline Estudos que utilizem como participantes atletas e desportistas profissionais. \\
\hline Publicações nos idiomas inglês, português ou espanhol. \\
\hline Publicação na íntegra online com acesso gratuito. \\
\hline \multicolumn{1}{c|}{ Justificativa de exclusão } \\
\hline Estudos que não se relacionam com habilidades sociais no esporte. \\
\hline Estudos que os participantes sejam amadores ou não atletas. \\
\hline Publicações que não apresentem técnicas ou instrumentos psicológicos em seu método. \\
\hline Publicação paga. \\
\hline
\end{tabular}

Fonte: Autores (2021).

Os artigos a serem considerados serão baseados no mnemônico PCC (População, Conceito e Contexto) anteriormente citado, nos idiomas português, espanhol e inglês. Sem delimitação de início que se estendam até 2021, pois utilizando artigos desse período, será composta uma análise mais completa na linha de pesquisa proposta, podendo assim realizar alguns comparativos de produções atuais com anteriores e admitindo tais idiomas é possível realizar análises de variadas realidades.

\subsection{Mapeamento da informação}

Para a análise e posterior síntese dos artigos será utilizado um formulário extração de dados (quadro 2), que constarão informações bibliográficas básicas, características dos estudos e informações que permitam descrever os principais aspectos sobre habilidades sociais no esporte.

O formulário de extração será preenchido pelos revisores de forma independente, as questões e dúvidas sobre as extrações serão tratadas por consenso entre os três revisores principais e, quando não resolvidos, serão discutidos com um quarto revisor.

As informações coletadas na fase de extração serão armazenadas em um banco de dados eletrônico criado no programa Microsoft Excel for Windows® versão 2019. Serão retirados alguns dados relativos aos artigos, informações como: título, nome dos autores e ano, objetivo do estudo, método (participantes da pesquisa, técnicas e instrumentos utilizados), principais achados e limitações. 
Quadro 2. Formulário de extração de dados.

\begin{tabular}{|c|c|}
\hline Informações extraídas & Descrição \\
\hline Autores & Sobrenome dos autores \\
\hline Ano & Ano da publicação \\
\hline Título & Título original da publicação \\
\hline Tipo de publicação & Tese, dissertação ou artigo \\
\hline Revista & Nome da revista \\
\hline \multicolumn{2}{|c|}{ Característica dos estudos } \\
\hline Objetivo & Descrever o objetivo principal do estudo \\
\hline \multirow{4}{*}{ Método } & Tipo de método: quantitativo ou qualitativo \\
\hline & $\begin{array}{l}\text { Participantes; idade, sexo, tempo de experiência no esporte, esporte e tipo } \\
\text { de modalidade (coletiva ou individual) }\end{array}$ \\
\hline & Técnicas e instrumentos utilizados \\
\hline & $\begin{array}{l}\text { Tipo de análise: comparação entre grupos, estatística descritiva, pré e pós } \\
\text { teste }\end{array}$ \\
\hline Principais achados & Descrever os principais resultados e conclusões do estudo \\
\hline Limitações & Descrever as limitações da pesquisa \\
\hline
\end{tabular}

Fonte: Autores (2021).

\subsection{Apresentação dos resultados}

A sumarização e apresentação dos resultados serão apresentadas em forma de quadro sintético e em formato descritivo conforme orientações do protocolo PRISMA - extensão para revisão de escopo (Tricco et al., 2018). O quadro sintético conterá um resumo dos estudos revisados de acordo com as informações do formulário de extração de dados, caracterizando os estudos e apresentando os construtos e as técnicas e instrumentos empregados para levantar, identificar ou avaliar habilidades sociais no contexto esportivo.

\subsection{Aspectos éticos}

Por se tratar de um estudo que utilizará informações bibliográficas disponíveis publicamente, não será necessária aprovação ética prévia por comitês de ética em pesquisa envolvendo seres humanos. Além disso, os autores dessa revisão não possuem vínculos que possam caracterizar potenciais conflitos de interesse. Os resultados dessa investigação serão publicados em periódico de acesso livre ou apresentados em eventos científicos relevantes.

Esse protocolo visa descrever e sistematizar as etapas metodológicas para realização de uma revisão de escopo das publicações sobre habilidades sociais no contexto esportivo, objetivando reduzir vieses na busca e seleção de referências, tornando claros e uniformes esses critérios entre os revisores. Destaca-se, ainda, que os dados compilados na revisão de escopo subsidiarão a construção de argumentos e informações para compreender como estão sendo identificados, levantados e/ou avaliadas as habilidades sociais no esporte. 


\section{Agradecimentos}

Agradecemos ao Centro Universitário Metropolitano da Amazônia (UNIFAMAZ) pelo fomento deste projeto de Iniciação científica, bem como ao Núcleo Gestor de Pesquisa e Extensão (NUPEX) pelo apoio acadêmico.

\section{Referências}

Andreoli, M. A., Oliveira, T. C., \& Fonteles, D. S. R. (2020). Um panorama das intervenções comportamentais para tratar estresse e ansiedade em atletas: revisão bibliográfica. Cadernos de Pós-Graduação em Distúrbios do Desenvolvimento, 20(1), 85-115. doi:10.47749/t/unicamp.2019.1095119

Aromataris E., \& Munn Z. (Eds.) (2020). JBI Manual for Evidence Synthesis. JBI. https://synt. hesismanual.jbi.global

Bartholomeu, D., Nunes, C. H. S. S., \& Machado, A. A. (2017). Traços de personalidade e habilidades sociais em universitários. Psico-USF, https://doi.org/10.1590/s1413-82712008000100006

Bessa, C., Hastie, P., Araújo, R., \& Mesquita, I. (2019). What do we know about the development of personal and social skills within the sport education model: A systematic review. Journal of Sports Science and Medicine, 18(4), 812-829.

Bessa, C., Hastie, P., Ramos, A., \& Mesquita, I. (2021). What actually differs between traditional teaching and sport education in students' learning outcomes? A critical systematic review. Journal of Sports Science and Medicine, 20(1), 110-125. doi:10.52082/jssm.2021.110

Braga, H. F., Furlanetto, D., \& Nazari, J. (2007). Paintball, estratégia e inteligência: uma alternativa para o esporte e o lazer. Revista Digital Deportiva, 3(4), $101-109$.

Bueno, J. M. H., Oliveira, S. M. S. S., \& Oliveira, J. C. S. (2011). Um estudo correlacional entre habilidades sociais e traços de personalidade. Psico-USF, 6(1), 31-38. doi:10.1590/s1413-82712001000100005

Camargo, T. P. P. D., Costa, S. P. V. D., Uzunian, L. G., \& Viebig, R. F. (2008). Vigorexia: revisão dos aspectos atuais deste distúrbio de imagem corporal. Revista Brasileira de Psicologia do Esporte, 2(1), 01-15.

Carder, S. L., Giusti, N. E., Vopat, L. M., Tarakemeh, A., Baker, J., Vopat, B. G., \& Mulcahey, M. K. (2020). The concept of sport sampling versus sport specialization: Preventing youth athlete injury: A systematic review and meta-analysis. American Journal of Sports Medicine, 48(11), $2850-2857$. doi:10.1177/0363546519899380

Cook, C. R., Gresham, F. M., Kern, L., Barreras, R. B., Thornton, S., \& Crews, S. D. (2008). Social skills training for secondary students with emotional and/or behavioral disorders: A review and analysis of the meta-analytic literature. Journal of Emotional and Behavioral Disorders, 16(3). doi:10.1177/1063426608314541

Cruz, D. R., \& Sehnem, S. B. (2015). Avaliação do repertório de habilidades sociais de crianças em um programa de educação pelo esporte. Pesquisa em Psicologia, 75-87.

Cunha, A. C. S., Moreira, I. D., \& Faria, G. N. (2021). Quando a psicologia entra em campo: uma revisão integrativa da literatura. Revista Artigos.Com, 25, e5482. Recuperado de https://acervomais.com.br/index.php/artigos/article/view/5482

Del Prette, Z. A. P., \& Del Prette, A. (2018). Competência social e habilidades sociais: Manual teórico-prático. Editora Vozes.

Del Prette, Z. A. P., \& Del Prette, A. (2017). Habilidades sociais e competência social para uma vida melhor. EdUFSCar. 10.7476/9786580216161

Feitosa, F. B., Matos, M. G., Del Prette, Z. A. P., \& Del Prette, A. (2005). Suporte social, nível socioeconômico e o ajustamento social e escolar de adolescentes portugueses. Temas em Psicologia, 13(2), 129-138.

Fernandes, M. G., Abreu, I. D. S., Alcantara, J., \& Nunes, S. A. N. (2019). Efeito isolado e combinado do sexo, tipo de esporte e experiência competitiva sobre as habilidades psicológicas. Educación Física y Ciencia, 21(2), e079. Recuperado de http://www.memoria.fahce.unlp.edu.ar/art_revistas/pr.10983/pr.10983.pdf

Filho, M. G. B., Ribeiro, L. C. S., \& García, F. G. (2005). Comparação de características da personalidade entre atletas brasileiros de alto rendimento e indivíduos não-atletas. Revista Brasileira de Medicina do Esporte, 11(2), 115-120. Recuperado de https://doi.org/10.1590/S1517-86922005000200004

Freita, L. C., Bandeira, M., Del Prette, A., \& Del Prette, Z. A. P. (2016). Comparando indicadores psicométricos de duas versões brasileiras do social skills rating system: Uma revisão da literatura. Psico-USF, 21(1), 25-36. Recuperado de https://doi.org/10.1590/1413-82712016210103

García-Coll, V., Graupera-Sanz, J. L., Ruiz-Pérez, L. M., \& Palomo-Nieto, M. (2013). Inteligencia emocional en el deporte: Validación española del Schutte Self Report Inventory (SSRI) en deportistas españoles. Cuadernos de Psicología Del Deporte, 13(1), 25-36.

Medeiros, C., \& Lacerda, A. (2017). Psicologia e esporte na atualidade: reflexões necessárias. Passavento.

Michael Normand, J., Wolfe, A., \& Peak, K. (2017). A review of early sport specialization in relation to the development of a young athlete. International Journal of Kinesiology and Sports Science, 5(2), 37-42. https://doi.org/10.7575/aiac.ijkss.v.5n.2p.37

Rocha, G. M., \& Monteiro, M. F. B. (2017). Programa de treinamento de habilidades sociais para a prática do futebol. Psicologia Argumento, 30(68), 63-74. https://doi.org/10.7213/rpa.v30i68.19561

Santos, A. A. A. Sisto, F. F., \& Martins, R. M. M. (2011). Estilos cognitivos e personalidade: um estudo exploratório de evidências de validade. Psico-USF, 8(1), 11-19. https://doi.org/10.1590/s1413-82712003000100003 
Research, Society and Development, v. 10, n. 14, e79101421469, 2021

(CC BY 4.0) | ISSN 2525-3409 | DOI: http://dx.doi.org/10.33448/rsd-v10i14.21469

Stefanello, J. M. F. (2007). Treinamento de competências psicológicas: Em busca da excelência esportiva. Manole.

Trevelin, F., \& Alves, C. F. (2019). Psicologia do esporte: revisão de literatura sobre as relações entre emoções e o desempenho do atleta. Psicologia Revista, 27, 545-562. https://doi.org/10.23925/2594-3871.2018v27i3p545-562

Tricco, A. C., Lillie, E., Zarin, W., O’Brien, K. K., Colquhoun, H., Levac, D., Moher, D., Peters, M. D. J., Horsley, T., Weeks, L., Hempel, S., Akl, E. A., Chang, C., McGowan, J., Stewart, L., Hartling, L., Aldcroft, A., Wilson, M. G., Garritty, C., \& Straus, S. E. (2018). PRISMA extension for scoping reviews (PRISMAScR): Checklist and explanation. In Annals of Internal Medicine, 169(7), 467-473. https://doi.org/10.7326/M18-0850

Weinberg, R. S., \& Gould, D. (2015). Fundamentos da Psicologia do Esporte e do Exercício (6th ed.). Artmed. 